\title{
Upregulation of miR-520b promotes ovarian cancer growth
}

\author{
RUI GUAN $^{1 *}$, SHENGYUN CAI $^{1 *}$, MINGJUAN SUN ${ }^{2 *}$ and MINGJUAN XU ${ }^{1}$ \\ ${ }^{1}$ Department of Obstetrics and Gynecology, Changhai Hospital, Second Military Medical University; \\ ${ }^{2}$ Department of Biochemistry and Molecular Biology, Second Military Medical University, Shanghai 200433, P.R. China
}

Received April 13, 2015; Accepted July 12, 2016

DOI: $10.3892 / \mathrm{ol} .2017 .6552$

\begin{abstract}
Ovarian cancer is the most common gynecological malignant cancer in female genitalia. Dysregulation or dysfunction of microRNAs (miRs) contribute to cancer development. The role of miR-520b in ovarian cancer remains unclear. The present study investigated the role of miR-520b in ovarian cancer and determined that the expression levels of miR-520b in ovarian cancer tissues and cell lines were upregulated. By contrast, reverse transcription-quantitative polymerase chain reaction and immunohistochemistry revealed that the mRNA and protein expression levels of ring finger protein 216 (RNF216) were downregulated in ovarian cancer, indicating that there was a negative correlation between miR-520b and RNF216. In miR-520b-knockdown cells, downregulation of miR-520b reduced cell proliferation, while upregulation of miR-520b promoted cell proliferation. In addition, RNF216 was predicted by TargetScanHuman and was observed to be targeted by miR-520b. In conclusion, the present data indicated that high expression of miR-520b in ovarian cancer promoted cell growth via RNF216.
\end{abstract}

\section{Introduction}

Ovarian cancer, together with endometrial cancer and cervical carcinoma, are the three most common gynecological malignant cancers in the female genital system (1). Ovarian cancer ranks the fifth of all female malignancies, causing 14,270 mortalities in the USA per year $(1,2)$. Management of ovarian cancer generally consists of surgery followed by chemotherapy (3-6). Although surgery and platinum-based chemotherapy currently provide the cornerstone of standard ovarian cancer management pathways, novel therapy strategies are urgently required to improve outcomes $(5,6)$. To

Correspondence to: Dr Mingjuan Xu, Department of Obstetrics and Gynecology, Changhai Hospital, Second Military Medical University, 168 Changhai Road, Shanghai 200433, P.R. China E-mail: cngreen785@163.com

${ }^{*}$ Contributed equally

Key words: miR-520b, ovarian cancer, RNF216, cancer growth develop effective ovarian cancer treatments, elucidation of the molecular pathogenesis of ovarian cancer is required.

MicroRNAs (miRNAs or miRs) are a class of small RNAs of $\sim 18-22$ nucleotides in length (7). miRNAs suppress protein expression by inhibiting messenger RNA (mRNA) translation or inducing mRNA degradation by binding to the 3'-untranslated region (3'-UTR) of their target mRNAs (7). Deregulation or dysfunction of miRNAs contribute to cancer development (7-12). A number of miRNAs have been identified as highly up- or downregulated in ovarian cancer (13-19). However, their roles in the pathogenesis of ovarian cancer remain unclear, and whether other miRNAs may be involved in the pathogenesis of ovarian cancer remains unknown.

Previous studies demonstrated that miR-520b was downregulated in breast cancer cells and contributed to the migration of highly metastatic breast cancer cells via a network that involves two target genes, hepatitis B virus X-interacting protein (HBXIP) and interleukin (IL)-8, in which HBXIP promotes cell migration by upregulation of IL-8, which is mediated by nuclear factor- $\kappa \mathrm{B}(20)$.

In the present study, the role of miR-520b in ovarian cancer was investigated. The data indicated that miR-520b promoted cellular proliferation, and suggested that the targeted gene of miR-520b may be ring finger protein 216 (RNF216). The present results may provide a novel therapeutic target for ovarian cancer.

\section{Materials and methods}

Cell culture and transfection. The human ovarian cancer cell lines SKOV3, Hey and OVCAR3 as well as the human embryonic kidney (HEK) 293 cell line were purchased from the Cell Bank of the Chinese Academy of Sciences (Shanghai, China). The cell lines were maintained in RPMI 1640 medium (Invitrogen; Thermo Fisher Scientific, Inc., Waltham, MA, USA) in the presence of $10 \%$ heat-inactivated fetal bovine serum (Biological Industries, Beit Haemek, Israel), 100 IU/ml penicillin and $100 \mathrm{ng} / \mathrm{ml}$ streptomycin in a humidified 5\% (v/v) atmosphere of $\mathrm{CO}_{2}$ at $37^{\circ} \mathrm{C}$. Transfection was performed using Lipofectamine 2000 reagent (Invitrogen; Thermo Fisher Scientific, Inc., Waltham, MA, USA) according to the manufacturer's protocol.

Patients. Surgical specimens from 30 ovarian cancer patients and matched tumor adjacent normal tissues were obtained postoperatively from April to June, 2008 from the Department of Obstetrics and Gynecology of Changhai Hospital, Second 


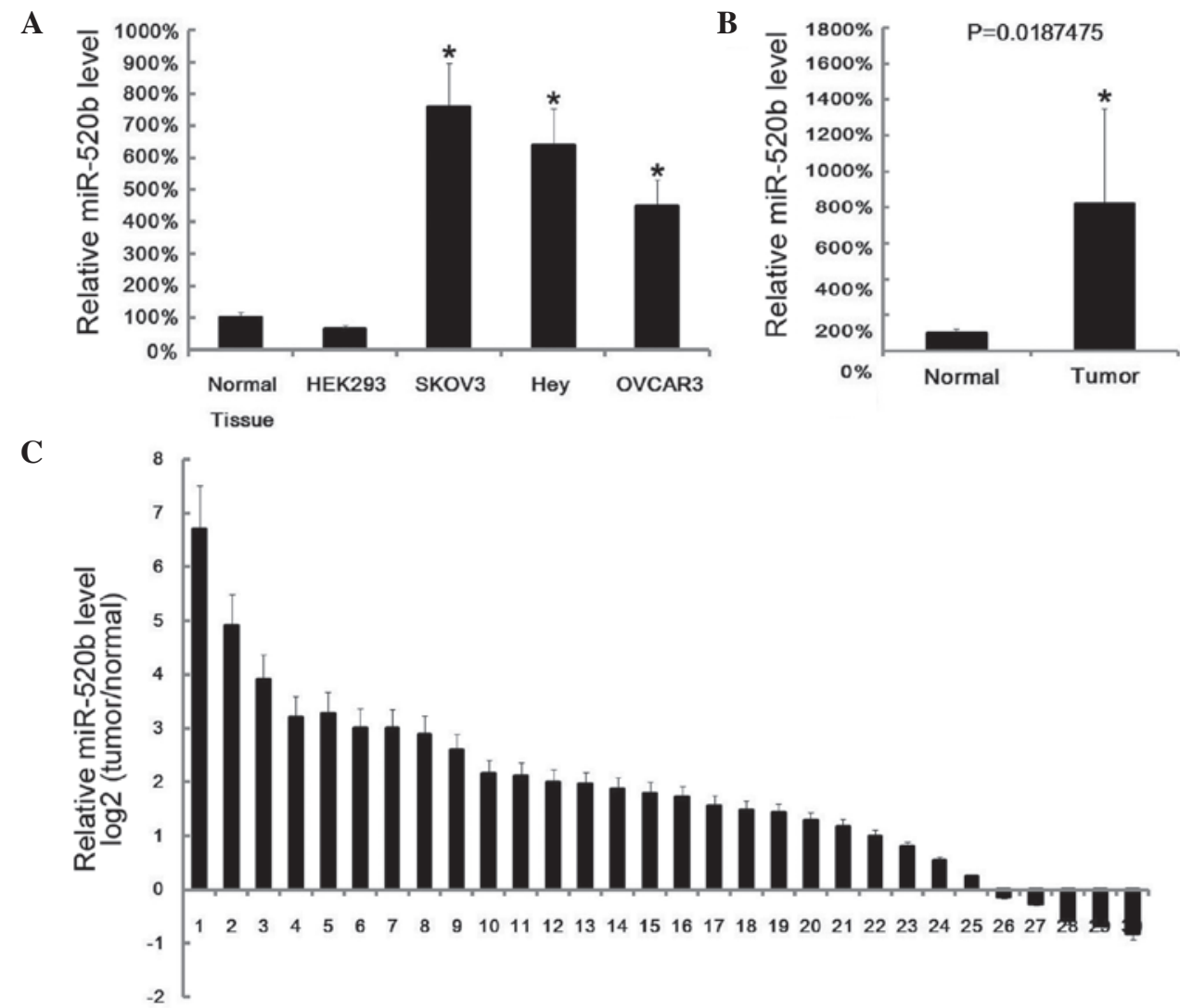

Figure 1. High expression of miR-520b in ovarian cancer tissues and ovarian cancer cell lines. (A) Following RNA extraction, miR-520b levels in normal tissues, HEK293 cells, and SKOV3, Hey and OVCAR3 cell lines were assayed by RT-qPCR. The miR-520b expression level in normal ovarian tissue was arbitrarily defined as $100 \%$. Data are represented as the mean $\pm \mathrm{SD}$ of three separate experiments. (B) The expression of miR-520b in ovarian cancer tissues and adjacent normal tissues was assayed by RT-qPCR. The miR-520b mean expression level in normal ovarian tissues was arbitrarily defined as $100 \%$. Data are represented as the mean $\pm \mathrm{SD}$ of 30 samples. (C) The miR-520b expression levels in ovarian cancer tissues and adjacent normal tissues were assayed by RT-qPCR. The differences in miR-520b expression between tumor tissues and adjacent normal tissues in each patient were compared. ${ }^{*} \mathrm{P}<0.05$ vs. control. miR, microRNA; HEK, human embryonic kidney; RT-qPCR, reverse transcription-quantitative polymerase chain reaction; SD, standard deviation.

Military Medical University (Shanghai, China). All diagnoses were based on pathological and/or cytological evidence. Tissues were obtained prior to chemotherapy and radiotherapy, and were immediately frozen and stored at $-80^{\circ} \mathrm{C}$ prior to being subjected to reverse transcription-quantitative polymerase chain reaction (RT-qPCR) analysis. Informed consent was obtained from each patient, and the study was approved by the Ethics Committee of the Second Military Medical University.

$R T$-qPCR. RT-qPCR analysis was performed on a 7500 Real-Time PCR System (Applied Biosystems; Thermo Fisher Scientific, Inc., Waltham, MA, USA). miR-520b expression was assessed using a mirVana ${ }^{\mathrm{TM}}$ qRT-PCR miRNA Detection kit (Ambion; Thermo Fisher Scientific, Inc., Austin, TX, USA). The primers were designed and synthesized by Shanghai Shenggong Biology Engineering Technology Service, Ltd. (Shanghai, China). U6 was used as an internal control. The relative messenger RNA (mRNA) levels of RNF216 were normalized to the mRNA levels of the housekeeping gene glyceraldehyde 3-phosphate dehydrogenase $\left(\right.$ GAPDH), and calculated by the $2-^{\Delta \Delta \mathrm{Cq}}$ method (21). The primers used were as follows: GAPDH forward, 5'-CCATGT TCGTCATGGG-TGTGAACCA-3' and reverse, 5'-GCCAGT AGAGGCAGGGATGATGTTG-3'; RNF216 forward, 5'-TCT TCCTGTCCTTAGTCGGGAG-3' and reverse, 5'-CTGGGC
AGCTGGTTTGATGA-3'; and U6 forward, 5'-GTGGAC CGCACAAGCTCGCT-3' and reverse, 5'-TTGTTGAACGGC ACTGTGTATAGCA-3'. A 2-step processwas used for denaturation, annealing and extension. i) The reaction temperature was increased to $95^{\circ} \mathrm{C}$ for $10 \mathrm{sec}$ to melt all dsDNA. ii) The temperature was lowered to $60^{\circ} \mathrm{C}$ for $30 \mathrm{sec}$ to promote primer binding to the template and subsequent elongation occurred due to sufficient activity of the DNA polymerase at this temperature. Steps 1-2 were repeated, for 40 cycles for GAPDH and RNF216, and for 45 cycles for miR-520b.

\section{3-(4,5-dimethylthiazol-2-yl)-2,5-diphenyltetrazolium bromide} (MTT) assay. For MTT assay, $5 \times 10^{3}$ cells/well were seeded in triplicate in a 96-well plate with complete growth medium. Cells were counted over 5 days using an MTT assay kit (Promega Corporation, Madison, WI, USA), as described previously $(10,22-25)$. The data were measured with a microtiter plate reader with 570-nm filters (Promega Corporation).

miRNAs antisense, miRNAs mimics, oligonucleotides and overexpression plasmids. miRNAs mimics (miR-520b mimics) and miRNAs antisense oligonucleotides (miR-520b ASO) were obtained from Shanghai GenePharma Co., Ltd.(Shanghai, China). miRNAs ASO, miRNAs mimics and negative control (NC) were transfected into cells at a concentration of $50 \mathrm{nM}$ 
A
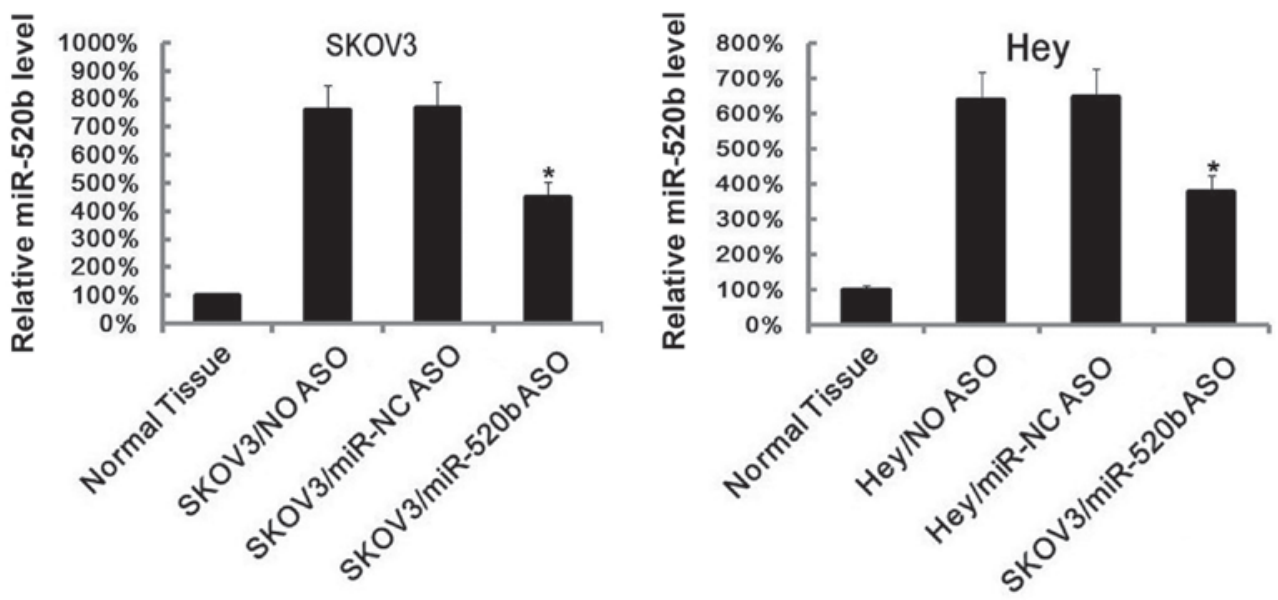

B
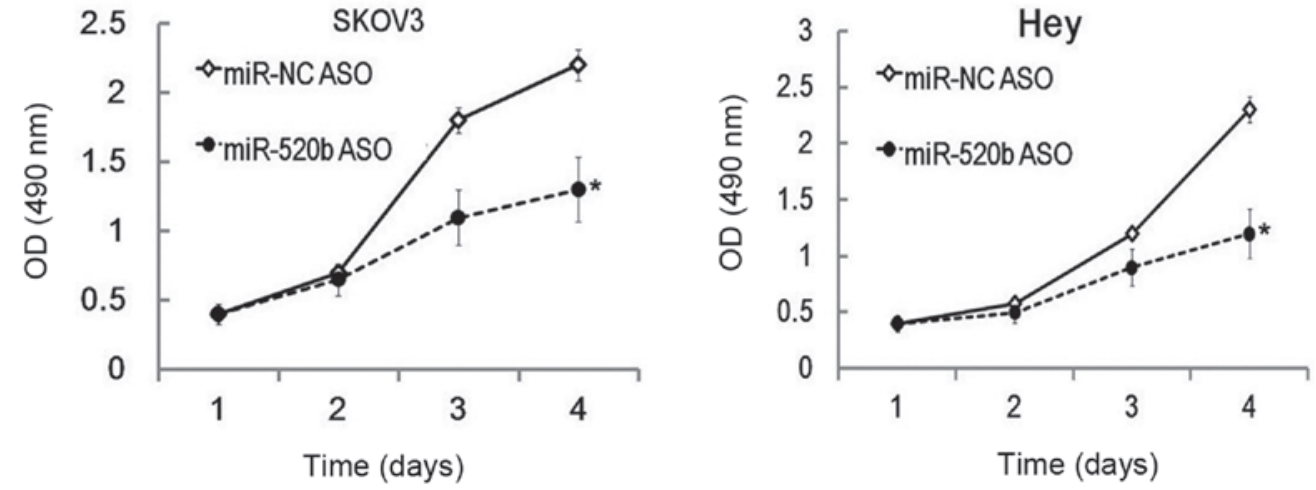

C

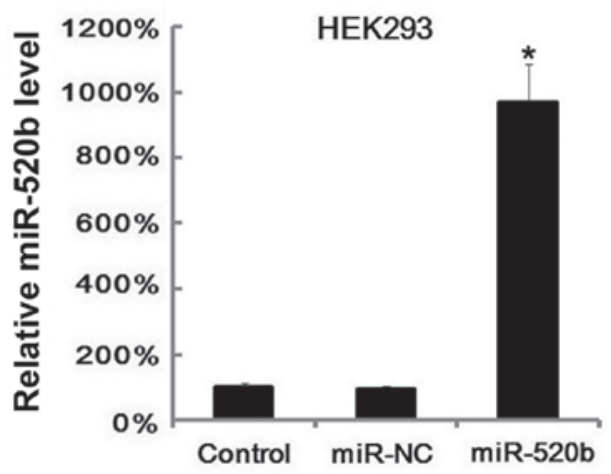

D

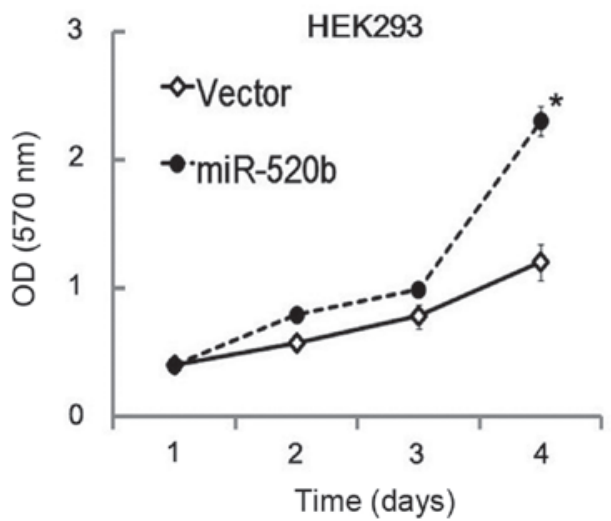

Figure 2. miR-520b downregulation reduced cell proliferation, while miR-520b upregulation promoted cell growth. (A) SKOV3 and Hey cells (6x105 cells/well) were transfected with miR-520b ASO or miR-NC ASO separately, and $48 \mathrm{~h}$ later, the expression of miR-520b was assayed by RT-qPCR. Data were normalized to U6 small nuclear RNA. The miR-520b expression level in ovarian cancer control samples was arbitrarily defined as $100 \%$. Data are represented as the mean \pm SD of three separate experiments. (B) Following miR-520b ASO transfection, cell proliferation was assayed with MTT at the indicted times. Data are represented as the mean \pm SD of three separate experiments. (C) HEK293 cells $\left(6 \times 10^{5}\right.$ cells/well) were transfected with miR-520b mimics, and $48 \mathrm{~h}$ later, the expression of miR-520b was assessed by RT-qPCR. Data were normalized to U6 small nuclear RNA. The miR-520b expression level in ovarian cancer control samples was arbitrarily defined as $100 \%$. Data are represented as the mean \pm SD of three separate experiments. (D) At $24 \mathrm{~h}$ after miR-520b mimics transfection, cell proliferation at different times was assayed with MTT. Data are represented as the mean \pm SD of three separate experiments. "P<0.05 vs. control. miR, microRNA; NC, negative control; ASO, antisense oligonucleotide; NO, untreated with ASO or non-specific oligonucleotide; OD, optical density; HEK, human embryonic kidney; RT-qPCR, reverse transcription-quantitative polymerase chain reaction; SD, standard deviation; MTT, 3-(4,5-dimethylthiazol-2-yl)2,5-diphenyltetrazolium bromide.

using Lipofectamine 2000 transfection reagent (Invitrogen; Thermo Fisher Scientific, Inc., Waltham, MA, USA) according to the manufacturer's protocol. At 48 or $72 \mathrm{~h}$ later, cells were collected for further experiments. The overexpression plasmid pcDNA3.1-RNF216 and the knockdown plasmid pcDNA3.1-RNF216 KD were constructed and confirmed by Shanghai Shenggong Biology Engineering Technology Service, Ltd.
miRNAs targets prediction. TargetScanHuman (http://www. targetscan.org/vert_61/) (26-29) was applied to identify the potential target of miR-520b.

RNF216 3'-UTR reporter analysis. The RNF216 3'-UTR reporter plasmids (pRL-RNF216) were constructed by Shanghai Shenggong Biology Engineering Technology Service, Ltd. Mutations in the miR-520b target regions of 
A

Wild type RNF216 3' UTR

hsa-miR-520b

Mutated RNE216 3' UTR

hsa-miR-520b

C

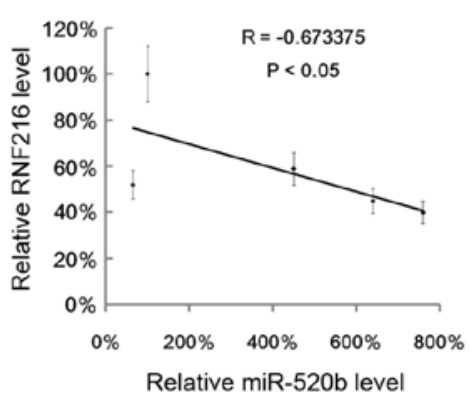

5' ... AgGaAUCUAGUaUUU-gCACUUAG. . .

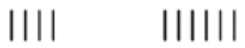

3' GGGAGAUUUUCCUUCGUGAAA

5' ... AgGaAUCUAGUAUUU-GCAAAAG...

IIII III

3' GGGAGAUUUUCCUUCGUGAAA

D

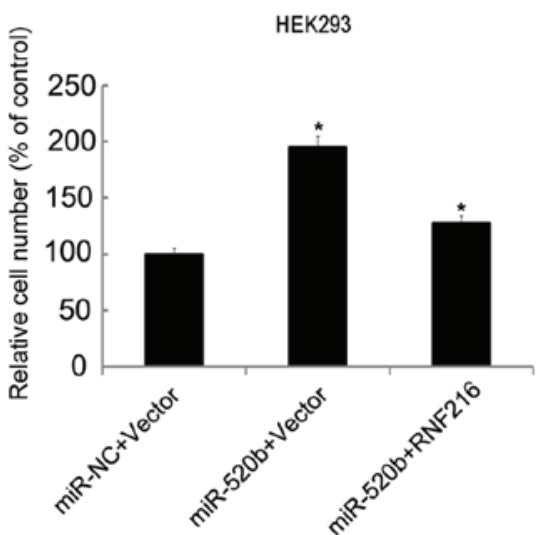

B

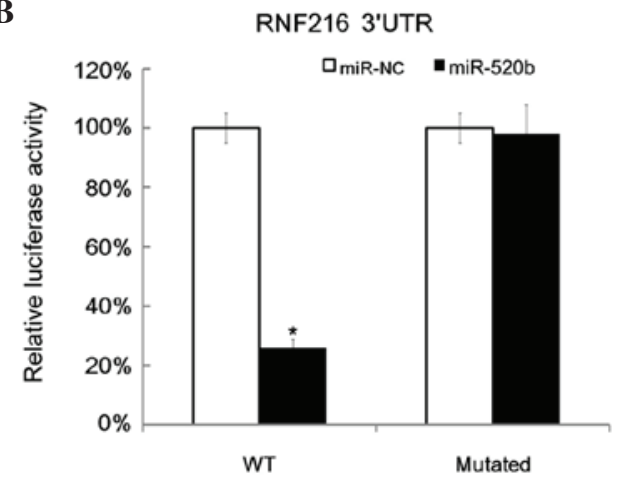

$\mathbf{E}$
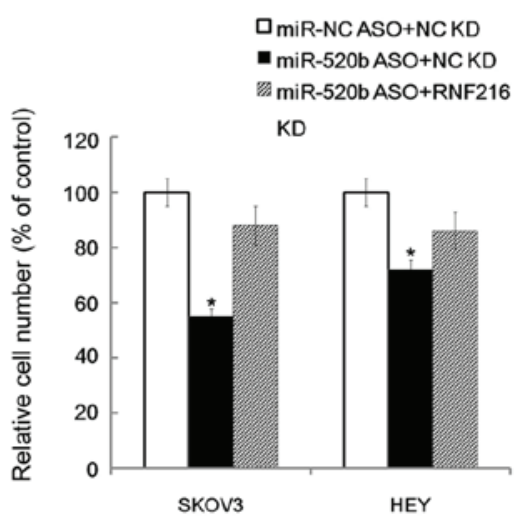

Figure 3. miR-520b-targeted genes prediction and validation. (A) Putative genes targeted by miR-520b were predicted by TargetScanHuman. The binding site of the putative targeted gene RNF216 and the mutated site of RNF216 were shown. (B) The RL reporter plasmids (RL-control, RL-RNF216 and RL-mutated RNF216) as well as miR-520b or miR-NC were co-transfected into HEK293 cells, along with a firefly luciferase reporter (pGL control) for normalization. Luciferase activities were measured after $48 \mathrm{~h}$, and the ratio of RL activity vs. firefly luciferase activity in the miR-520b-treated group was calculated and compared with that in the in miR-NC group (which was arbitrary defined as $100 \%$ ). Data are represented as the mean \pm SD of three separate experiments. (C) Inverse correlation between miR-520b and RNF216 levels in ovarian cancer tissues ( $=5$ ). Statistical analysis was performed using Pearson's correlation coefficient. (D) HEK293 cells (6x10 cells/well) were transfected with an RNF216 overexpression plasmid (pcDNA3.1-RNF216), and 12 h later, the cells were transfected with miR-520b mimics. After $12 \mathrm{~h}$, cell proliferation was evaluated by MTT assay. The relative cell number in the miR-NC + vector (which served as NC) group was defined as 100\%. (E) SKOV3 and Hey cells (6x10 cells/well) were transfected with pcDNA3.1-RNF216 knockdown plasmid, followed by transfection with miR-520b ASO. Cell proliferation was evaluated by MTT assay $24 \mathrm{~h}$ later. The relative cell number in the miR-NC ASO + empty vector (which served as NC) group was defined as $100 \%$. ' $\mathrm{P}<0.05$ vs. control. RNF216, ring finger protein 216; UTR, untranslated region; hsa, Homo sapiens; miR, microRNA; NC, negative control; ASO, antisense oligonucleotide; WT, wild type; HEK, human embryonic kidney; KD, knowckdown; RL, Renilla luciferase; MTT, 3-(4,5-dimethylthiazol-2-yl)-2,5-diphenyltetrazolium bromide.

the RNF216 3'-UTR were generated using QuikChange Multi Site-Directed Mutagenesis kit (Stratagene; Agilent Technologies, Inc., Santa Clara, CA, USA). Renilla luciferase reporter plasmids $(3.6 \mathrm{fmol})$ and pGL3-control (500 ng used for normalization; Promega Corporation) were transfected with Lipofectamine 2000 (Invitrogen; Thermo Fisher Scientific, Inc., Waltham, MA, USA) into HEK293 $\left(6 \times 10^{4}\right.$ cells/well). Cells were collected after $48 \mathrm{~h}$ for analysis using the Dual-Luciferase ${ }^{\circledR}$ Reporter Assay System (Promega Corporation) (30). The sequence of the primers used for cloning RNF216 3'UTR were as follows: Forward, 5'-CGACGCGTGGAGGGCCAGATGTGCCCATC-3'; and reverse, 5'-GGGTTTAAACATGTTTAAAATTCTGAT GTCATTTATTGG-3'.

Immunohistochemistry (IHC). IHC staining was performed as described previously (31). Briefly, $4-\mu \mathrm{m}$ thick sections were cut, and an anti-RNF216 antibody (cat. no. HPA018955; 1:1,000; Sigma-Aldrich, St. Louis, MO, USA) was applied. Subsequent counterstaining was performed with hematoxylin. The immunostaining results for RNF216 were evaluated using a semi-quantitative scoring system as described previously (32), which calculated the staining intensity and the percentage of positive cells. IHC staining was scored according to the following criteria:,$- 0-10 \%$ of the nucleated cells were stained;,$+ 10-40 \%$ of the nucleated cells were stained; $++; 40-70 \%$ of the nucleated cells were stained; and ,$+++ 70-100 \%$ of the nucleated cells were stained. RNF216 expression was considered to be observed when the score was $\geq+$. Alternatively, the IHC score of RNF216 expression was - to + and ++ to +++ , which represented low and high expression, respectively.

Statistical analysis. Data were presented as the mean \pm standard deviation from $\geq 3$ independent experiments. The differences between groups were analyzed using two-tailed Student's $t$ test when only two groups were compared. The differences between groups were analyzed using analysis of variance when $\geq 3$ groups were compared. Correlation analysis was performed by two-tailed Person's correlation coefficient analysis. Patients survival was determined by Kaplan-Meier analysis. Statistical analyses were performed 
A
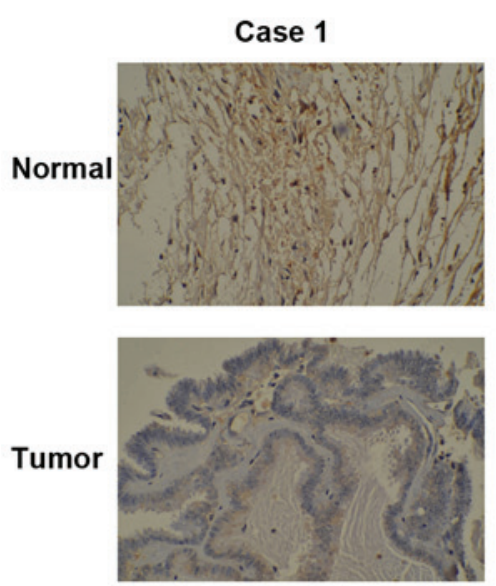

B

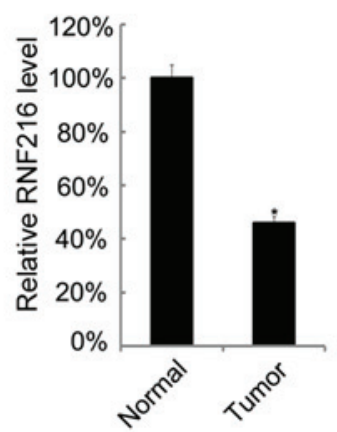

Case 3
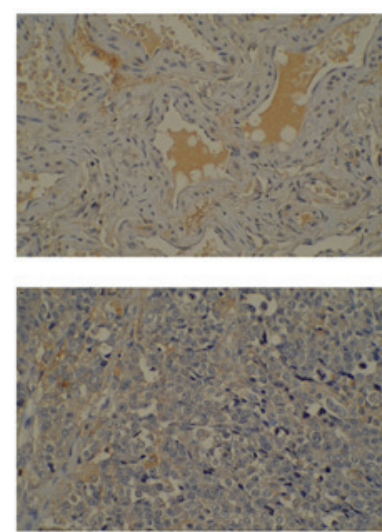

Case 4
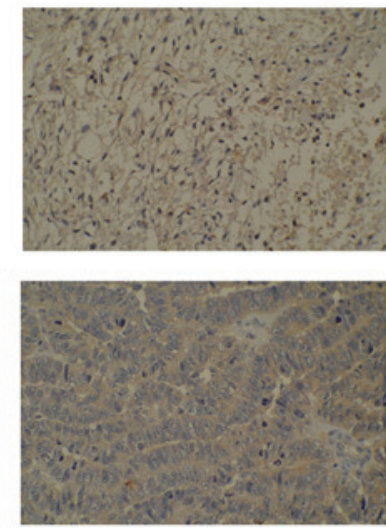

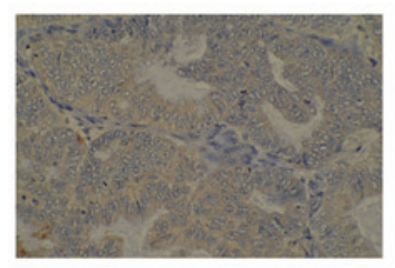

C

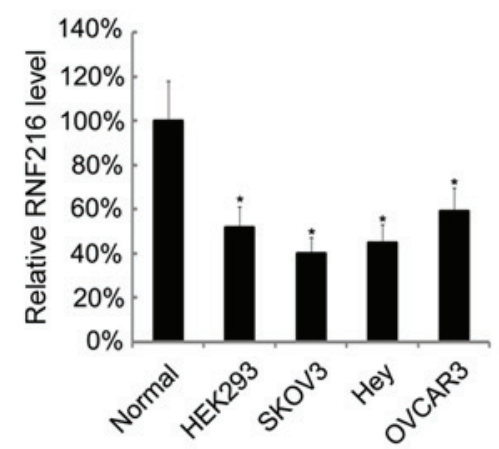

Figure 4. Low protein level and mRNA expression of RNF216 in ovarian cancer. (A) Representative images of RNF216 immunohistochemistry staining in ovarian cancer tissues and matched normal adjacent tissues (magnification, x100). (B) The mRNA levels of RNF216 in 30 ovarian cancer tissues were assayed by RT-qPCR. The mean RNF216 expression level in matched normal ovarian tissue was arbitrarily defined as $100 \%$. Data are represented as the mean \pm SD of the mRNA level of RNF216 in 30 ovarian cancer tissues (C) The mRNA levels of RNF216 in ovarian cancer cell lines were assayed by RT-qPCR. The RNF216 expression level in normal ovarian tissue was arbitrarily defined as $100 \%$. Data are represented as the mean $\pm \mathrm{SD}$ of three separate experiments. $\mathrm{P}<0.05$ vs. control. mRNA, messenger RNA; miR, microRNA; RNF216, ring finger protein 216; HEK, human embryonic kidney; SD, standard deviation; RT-qPCR, reverse transcription-quantitative polymerase chain reaction.

using SPSS software version 17.0 (SPSS, Inc., Chicago, IL, USA). $\mathrm{P}<0.05$ was considered to indicate a statistically significant difference.

\section{Results}

High expression of miR-520b in ovarian cancer. miR-520b expression was examined in human ovarian cancer cell lines (SKOV3, Hey and OVCAR3) and ovarian cancer tissues by RT-qPCR. It was observed that miR-520b exhibited high expression levels in ovarian cancer cell lines and ovarian cancer tissues (Fig. 1A and B). In total, 30 pairs of ovarian cancer tissues and matched adjacent normal tissues were collected from 30 ovarian cancer patients, and it was observed that, in 25 pairs, miR-520b expression was higher than in matched normal tissues (Fig. 1C).

Downregulation of miR-520b inhibits cells proliferation, while upregulation of miR-520b promotes cells proliferation. To investigate the role of miR-520b in ovarian cancer, miR-520b expression was suppressed in SKOV3 and Hey cells by ASO transfection. At $48 \mathrm{~h}$ after transfection, the suppressed miR-520b levels in SKOV3 and Hey cells were confirmed by RT-qPCR (Fig. 2A). Next, cell proliferation was assayed by MTT analysis, and it was observed that downregulation of miR-520b inhibited cell proliferation (Fig. 2B). Subsequently,
miR-520b was overexpressed by transfection of miR-520b mimics into HEK293 cells (Fig. 2C). Similarly, 48 h later, cell proliferation was evaluated by MTT analysis, and it was observed that overexpression of miR-520b promoted cell growth (Fig. 2D).

RNF216 is targeted by miR-520b. The potential target genes of miR-520b were explored using TargetScanHuman software, which predicted $\sim 884$ genes (data not shown). Of these, the presented study focused on an unreported gene, RNF216. The binding wild type (WT) and mutant sites in RNF216 are indicated in Fig. 3A. The WT 3'-UTR of RNF216 and its mutated version were cloned into luciferase reporter plasmids. miR-520b and the reporter plasmids were co-transfected into HEK293 cells. The results revealed that, in WT 3'-UTR, miR-520b reduced the luciferase activity, while in the mutated version, the difference between miR-NC and miR-520b was not significant ( $\mathrm{P}>0.05$; Fig. 3B).

Then, the correlation between miR-520b level and RNF216 in ovarian cancer tissues was explored. Pearson's correlation coefficient analysis revealed that RNF216 was inversely correlated with miR-520b level in ovarian cancer tissues (Fig. 3C). Next, miR-520b mimics and RNF216 overexpression plasmid were co-transfected into HEK293 cells. RNF216 overexpression partly reduced the cell proliferation effect of miR-520b (Fig. 3D). As expected, RNF216 downregulation 
partly reduced the growth inhibitory effect of miR-520b ASO in SKOV3 and Hey cells (Fig. 3E).

Low expression of RNF 216 in ovarian cancer. Next, the mRNA and protein levels of RNF216 in ovarian cancer tissues and cell lines were examined. There were lower levels of RNF216 in ovarian cancer tissues than in normal ovarian tissues. Four representative cases are shown in Fig. 4A. The mean levels of RNF216 in ovarian cancer tissues were lower than those in matched tumor adjacent normal tissues (Fig. 4B). RNF216 expression in ovarian cancer cell lines was also assessed, and RNF216 displayed lower expression levels in ovarian cancer cell lines (Fig. 4C).

\section{Discussion}

In the present study, high expression of miR-520b and low expression of RNF216 was detected in ovarian cancer tissues, and RNF216 was observed to be inversely correlated with miR-520b level. In cell experiments, the miR-520b level was regulated by miRNAs mimics or ASO transfection, and it was demonstrated that downregulation of miR-520b inhibited cell proliferation, whereas upregulation of miR-520b promoted cell proliferation. RNF216 3'-UTR reporter analysis revealed that miR-520b could target RNF216 in vitro. These data led to the conclusion that high expression of miR-520b in ovarian cancer promoted cell growth via RNF216. To the best of our knowledge, the present study is the first to demonstrate that miR-520b targets RNF216 in ovarian cancer.

The present data indicated that the expression of miR-520b was higher in ovarian cancer tissues than that in normal control tissues. However, a previous study reported that miR-520b was downregulated in breast cancer cells, which contributed to the migration of these cells (20). In hepatocellular carcinoma, miR-520b was downregulated in tumor tissues and hepatoma cell lines, and ectopic expression of miR-520b inhibited the growth of hepatoma cells in vitro and in vivo (33). This discrepancy may be explained by the genes targeted by miR-520b. In breast cancer cells, miR-520b targeted HBXIP and IL-8, while in hepatocellular carcinoma, miR-520b targeted mitogenactivated protein kinase kinase kinase 2 (MEKK2) and cyclin D1 (33). The present study demonstrated that miR-520b targeted RNF216. Whether miR-520b targeted HBXIP, IL-8, MEKK2 and cyclin D1 in ovarian cancer requires further investigation.

The bioinformatics algorithm used in the present study predicted 884 genes potentially targeted by miR-520b, including RNF216. There were two reasons for selecting RNF216 for further investigation. One is that RNF216 has not been reported yet in ovarian cancer. The other is that data from the preliminary test (microarray) revealed low RNF216 expression in ovarian cancer.

In conclusion, the present data demonstrated the role of miR-520b in ovarian cancer, and suggested that high expression of miR-520b in ovarian cancer promoted cell growth via RNF216. The present report is a preliminary study to outline the function of miR-520b. Despite certain discrepancy with the literature, the findings from the current study may provide the basis for future cancer studies.

\section{References}

1. Siegel R, Ma J, Zou Z and Jemal A: Cancer statistics, 2014. CA Cancer J Clin 64: 9-29, 2014.

2. Zhao YN, Chen GS and Hong SJ: Circulating MicroRNAs in gynecological malignancies: From detection to prediction. Exp Hematol Oncol 3: 14, 2014.

3. Rooth C: Ovarian cancer: Risk factors, treatment and management. Br J Nurs 22: S23-S30, 2013.

4. Bristow RE, Tomacruz RS, Armstrong DK, Trimble EL and Montz FJ: Survival effect of maximal cytoreductive surgery for advanced ovarian carcinoma during the platinum era: A meta-analysis. J Clin Oncol 20: 1248-1259, 2002.

5. Winter-Roach BA, Kitchener HC and Lawrie TA: Adjuvant (post-surgery) chemotherapy for early stage epithelial ovarian cancer. Cochrane Database Syst Rev 3: CD004706, 2012.

6. Winter-Roach BA, Kitchener HC and Dickinson HO: Adjuvant (post-surgery) chemotherapy for early stage epithelial ovarian cancer. Cochrane Database Syst Rev: CD004706, 2009.

7. Ambros V: The functions of animal microRNAs. Nature 431: 350-355, 2004.

8. Garzon R, Calin GA and Croce CM: MicroRNAs in cancer. Annu Rev Med 60: 167-179, 2009.

9. Hou J, Lin L, Zhou W, Wang Z, Ding G, Dong Q, Qin L, Wu X, Zheng Y, Yang Y, et al: Identification of miRNomes in human liver and hepatocellular carcinoma reveals miR-199a/b-3p as therapeutic target for hepatocellular carcinoma. Cancer Cell 19: 232-243, 2011.

10. Li D, Liu X, Lin L, Hou J, Li N, Wang C, Wang P, Zhang Q, Zhang P, Zhou W, et al: MicroRNA-99a inhibits hepatocellular carcinoma growth and correlates with prognosis of patients with hepatocellular carcinoma. J Biol Chem 286: 36677-36685, 2011.

11. Mendell JT and Olson EN: MicroRNAs in stress signaling and human disease. Cell 148: 1172-1187, 2012.

12. Croce CM: Causes and consequences of microRNA dysregulation in cancer. Nat Rev Genet 10: 704-714, 2009.

13. Iorio MV, Visone R, Di Leva G, Donati V, Petrocca F, Casalini P, Taccioli C, Volinia S, Liu CG, Alder H, et al: MicroRNA signatures in human ovarian cancer. Cancer Res 67: 8699-8707, 2007.

14. Resnick KE, Alder H, Hagan JP, Richardson DL, Croce CM and Cohn DE: The detection of differentially expressed microRNAs from the serum of ovarian cancer patients using a novel real-time PCR platform. Gynecol Oncol 112: 55-59, 2009.

15. Chung YW, Bae HS, Song JY, Lee JK, Lee NW, Kim T and Lee KW: Detection of microRNA as novel biomarkers of epithelial ovarian cancer from the serum of ovarian cancer patients. Int J Gynecol Cancer 23: 673-679, 2013.

16. Shapira I, Oswald M, Lovecchio J, Khalili H, Menzin A, Whyte J, Dos Santos L, Liang S, Bhuiya T, Keogh M, et al: Circulating biomarkers for detection of ovarian cancer and predicting cancer outcomes. Br J Cancer 110: 976-983, 2014.

17. Suryawanshi S, Vlad AM, Lin HM, Mantia-Smaldone G, Laskey R, Lee M, Lin Y, Donnellan N, Klein-Patel M, Lee T, et al: Plasma microRNAs as novel biomarkers for endometriosis and endometriosis-associated ovarian cancer. Clin Cancer Res 19: 1213-1224, 2013.

18. Zheng H, Zhang L, Zhao Y, Yang D, Song F, Wen Y, Hao Q, Hu Z, Zhang W and Chen K: Plasma miRNAs as diagnostic and prognostic biomarkers for ovarian cancer. PLoS One 8: e77853, 2013.

19. Kan CW, Hahn MA, Gard GB, Maidens J, Huh JY, Marsh DJ and Howell VM: Elevated levels of circulating microRNA-200 family members correlate with serous epithelial ovarian cancer. BMC Cancer 12: 627, 2012.

20. Hu N, Zhang J, Cui W, Kong G, Zhang S, Yue L, Bai X, Zhang Z, Zhang W, Zhang $X$ and Ye L: miR-520b regulates migration of breast cancer cells by targeting hepatitis B X-interacting protein and interleukin-8. J Biol Chem 286: 13714-13722, 2011.

21. Livak KJ and Schmittgen TD: Analysis of relative gene expression data using real-time quantitative PCR and the 2(-Delta Delta C(T)) Method. Methods 25: 402-408, 2001.

22. van Meerloo J, Kaspers GJ and Cloos J: Cell sensitivity assays: The MTT assay. Methods Mol Biol 731: 237-245, 2011.

23. Han ZB, Yang Z, Chi Y, Zhang L, Wang Y, Ji Y, Wang J, Zhao H and Han ZC: MicroRNA-124 suppresses breast cancer cell growth and motility by targeting CD151. Cell Physiol Biochem 31: 823-832, 2013.

24. Song B, Zhang C, Li G, Jin G and Liu C: MiR-940 inhibited pancreatic ductal adenocarcinoma growth by targeting MyD88. Cell Physiol Biochem 35: 1167-1177, 2015. 
25. Wu N, Zhang C, Bai C, Han YP and Li Q: MiR-4782-3p inhibited non-small cell lung cancer growth via USP14. Cell Physiol Biochem 33: 457-467, 2014

26. Lewis BP, Burge CB and Bartel DP: Conserved seed pairing, often flanked by adenosines, indicates that thousands of human genes are microRNA targets. Cell 120: 15-20, 2005.

27. Friedman RC, Farh KK, Burge CB and Bartel DP: Most mammalian mRNAs are conserved targets of microRNAs. Genome Res 19: 92-105, 2009.

28. Grimson A, Farh KK, Johnston WK, Garrett-Engele P, Lim LP and Bartel DP: MicroRNA targeting specificity in mammals: Determinants beyond seed pairing. Mol Cell 27: 91-105, 2007.

29. Garcia DM, Baek D, Shin C, Bell GW, Grimson A and Bartel DP: Weak seed-pairing stability and high target-site abundance decrease the proficiency of 1sy- 6 and other microRNAs. Nat Struct Mol Biol 18: 1139-1146, 2011.
30. Grentzmann G, Ingram JA, Kelly PJ, Gesteland RF and Atkins JF: A dual-luciferase reporter system for studying recoding signals. RNA 4: 479-486, 1998.

31. Xu XS, Wang L, Abrams J and Wang G: Histone deacetylases (HDACs) in XPC gene silencing and bladder cancer. J Hematol Oncol 4: 17, 2011.

32. Lv T, Yuan D, Miao X, Lv Y, Zhan P, Shen X and Song Y: Over-expression of LSD1 promotes proliferation, migration and invasion in non-small cell lung cancer. PLoS One 7: e35065, 2012.

33. Zhang W, Kong G, Zhang J, Wang T, Ye L and Zhang X: MicroRNA-520b inhibits growth of hepatoma cells by targeting MEKK2 and cyclin D1. PLoS One 7: e31450, 2012. 